\title{
A bright future for colloidal quantum dot lasers
}

Pieter Geiregat $\mathbb{D}^{1,2}$, Dries Van Thourhout ${ }^{2,3}$ and Zeger Hens ${ }^{1,2}$

Since they were first synthesized nearly 30 years ago, colloidal quantum dots (QDs) have attracted much attention as an enabling material for solution-processed opto-electronics $^{1,2}$. QDs offer seamless tunability of the optical bandgap from the UV to the mid-infrared (IR), are fit for substrate-independent and cost-effective processing, and have the prospect of long-term stability inherent to inorganic materials. Given these advantages, QDs quickly took a firm position in the optoelectronic materials community. As of today, QDs find applications in diverse fields such as biolabeling, displays, lighting, sensing, and solar energy conversion ${ }^{3}$, applications that all rely on the spectrally broad absorption or the highly efficient and spectrally narrow photoluminescence of QDs.

Nearly two decades ago, Klimov and coworkers demonstrated net stimulated emission and amplified spontaneous emission by CdSe-based QDs, a result that opened up the exciting prospect of fabricating QD-based lasers ${ }^{3,4}$. Lasers are ubiquitous in modern society as sources of spatio-temporal coherent and quasimonochromatic light. Lasers find applications in consumer products, telecommunications, healthcare, imaging, and so on and enable fundamental research in increasingly diverse fields. Even so, laser fabrication remains challenging due to the stringent requirements on the active light-amplifying medium, the pump mechanisms and the need for a high-quality feedback structure or optical cavity. Current opto-electronic technology heavily relies on epitaxially grown semiconductors, a materials platform that is, however, limited by its high cost, limited spectral tunability and the need for rigid substrate-

\footnotetext{
Correspondence: Pieter Geiregat (pieter.geiregat@ugent.be)

${ }^{1}$ Physics and Chemistry of Nanostructures Group, Department of Chemistry, Ghent University, 9000 Gent, Belgium

${ }^{2}$ Centre for Nano and Bio-photonics, Ghent University, 9000 Gent, Belgium Full list of author information is available at the end of the article.
}

mediated growth. Given the current needs for lasers on flexible substrates ${ }^{5}$ or as disposable, low-cost appliances, such as a lab-on-a-chip ${ }^{6}$, this creates an opportunity for QDs to revolutionize the field of laser-based opto-electronics, much as these materials currently do for the growing markets of displays and lighting.

Lasing action requires that the roundtrip gain through stimulated emission in the cavity exceeds the losses due to re-absorption in the active medium and transmission through the cavity end mirrors, see Fig. 1a. Characterizing a QD by its two band-edge states, this roundtrip gain in a QD-laser is provided by stimulated emission across the band-edge transition. Building on the initial models of Klimov et al. ${ }^{3}$, one can propose an intuitive mathematical formulation of this 2-level model depicted in Fig. 1a that captures the essence of attaining net optical gain in a colloidal QD ensemble ${ }^{7}$. We consider a 2-level system with degeneracies $\left(g_{e}, g_{h}\right)$ for the electron/hole levels involved in the gain transition and linearly increasing spectral shifts of multi-excitons $\delta_{N}=N \delta_{E}$, where $\delta_{E}$ is the biexciton-exciton shift and $N$ the number of excitons. This brings us to the following expressions for the absorbance at an energy $E$ of unexcited QDs and QDs excited with $N_{e}$ electrons and $N_{h}$ holes:

- Absorbance of unexcited dots: $A_{0}(E)$

- Absorbance of QDs with $\left(N_{e}, N_{h}\right)$ (electrons, holes):

$$
A^{N}(E)=\left(1-\frac{N_{e}}{g_{e}}\right)\left(1-\frac{N_{h}}{g_{h}}\right) A_{0}\left(E-N \delta_{E}\right)
$$

- Stimulated emission of QDs with $\left(N_{e}, N_{h}\right)$ (electrons, holes):

$$
G^{N}(E)=\frac{N_{e}}{g_{e}} \frac{N_{h}}{g_{h}} A_{0}\left(E-(N-1) \delta_{E}\right)
$$

Note that optical excitation usually yields $N_{e}=N_{h}$, but special cases, such as doped QDs or trap-state gain, allow 

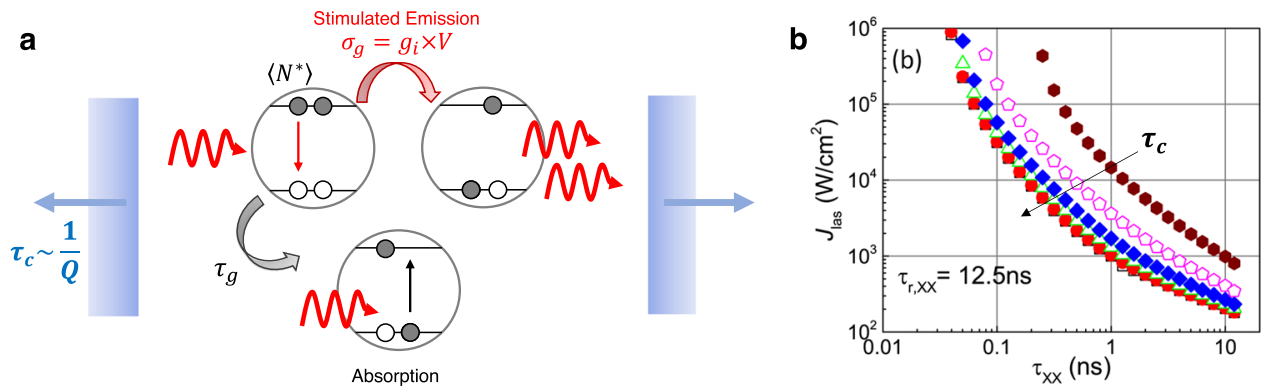

Fig. 1 Colloidal quantum dots (QDs) as optical gain material. a Typical loss channels for population inversion through bi-exciton Auger recombination, affecting the inversion lifetime $\tau_{\mathrm{g}}$ and photon loss through a finite photon-cavity lifetime $\left(\tau_{c}\right)$. (b) Calculated CW pump threshold as a function of the biexciton Auger lifetime $T_{x x}$ (horizontal axis) and cavity lifetimes varying (colors) from 1 ps to $1 \mathrm{~ns}$, adapted from Park et al. ${ }^{11}$

for $N_{e} \neq N_{h}$. In summary, we can write the absorbance $A$ as:

$$
A(E)=P(0) A_{0}(E)+\sum_{N \geq 1} P(N)\left(A^{N}(E)-G^{N}(E)\right)
$$

where $P(N)$ is the Poisson probability to have $N$ excitations in a dot given an average occupation of $\langle N\rangle$ : $P(N)=\frac{e^{-\langle N\rangle}\langle N\rangle^{N}}{N !}$. For example, one can show that for Cdbased QDs, $A$ turns negative if $\langle N\rangle$ excitations in a dot given an average exceeds unity, where the exact value depends on the assumed degeneracies of the hole levels involved ${ }^{7,8}$.

Building on this two-state model, net stimulated emission by an ensemble of QDs is typically summarized by three descriptors:

- The threshold occupation $\left\langle N^{*}\right\rangle$, which is the minimum number of electron-hole pairs QDs must hold on average to attain net stimulated emission.

- The inversion lifetime $\tau_{g}$, which is the time a state of net stimulated emission is preserved after pulsed photo-excitation.

- The material gain $g_{i}$, which is the gain, measured in $\mathrm{cm}^{-1}$, provided by a fictitious ensemble of QDs packed with a QD volume fraction of unity. It can be translated to device scenarios using the volume fraction and optical mode confinement of the QD layer in a laser cavity ${ }^{9}$.

Since the discovery of light amplification by QDs ${ }^{4}$, it was realized that net stimulated emission requires biexciton states, i.e., QDs containing two electron-hole pairs. Such states suffer from fast, non-radiative Auger recombination (see Fig. 1a), which limits $\tau_{g}$ to a few tens of ps in the case of bare CdSe QDs ${ }^{4,8}$. Because the threshold pump power $P_{\text {th }}$ required to achieve lasing action in a lossless cavity under steady-state conditions scales with the ratio $\left\langle N^{*}\right\rangle / \tau_{g}$, suppressing this fast Auger recombination was seen as a first step to achieve QD-lasers. By introducing $\mathrm{CdSe} / \mathrm{CdS}$ core/shell QDs with a smooth, alloyed interface, Garcia-Santamaria et al. were able to slow down the Auger process by orders of magnitude ${ }^{10}$ and render the Auger loss channel nearly ineffective ${ }^{3}$. Depending on the quality factor of the cavity-or the cavity lifetimePark et al. ${ }^{11}$ predicted that Auger lifetimes of $\sim 1 \mathrm{~ns}$ should result in CW pump thresholds of $1-10 \mathrm{~kW} / \mathrm{cm}^{2}$ (see Fig. 1b). The marked dependence of the lasing threshold on the lifetimes of photons in the photonic cavity indicates that QD laser progress will be closely connected to both materials and photonic engineering.

As CdSe/CdS QDs can have a material gain up to several $1000 \mathrm{~cm}^{-1} 7$, these core/shell QDs make an excellent model system to explore and improve the performance of QD-based lasers. This point has become evident in the past few years through the demonstration of applicationrelevant excitation schemes, such as electrical ${ }^{12}$ and continuous-wave operation ${ }^{13}$ and the demonstration of integrated QD lasers ${ }^{9}$. These milestones set the scene for a reflection on the future of QD lasers. In this short perspective, we therefore first address late breakthroughs in more detail in terms of both materials and device engineering, and we provide a set of possible future research directions to extend both the functionality and performance of QD lasers. Finally, we discuss several applications for which QD lasers can make a difference, an overview highlighting that QD lasers have reached a tipping point to become a commercially viable technology in the years to come.

\section{Key advances made}

Advances in QD lasing technology go hand-in-hand with improvements of QD properties in general and the development of reliable QD process technology. We accordingly split the breakthrough results of the past 5 years in two classes. On the one hand, proof-of-concept demonstrations have appeared involving room-temperature continuouswave, electrically pumped and on-chip lasing based on clever engineering around the $\mathrm{CdSe} / \mathrm{CdS}$ model QDs. On the 


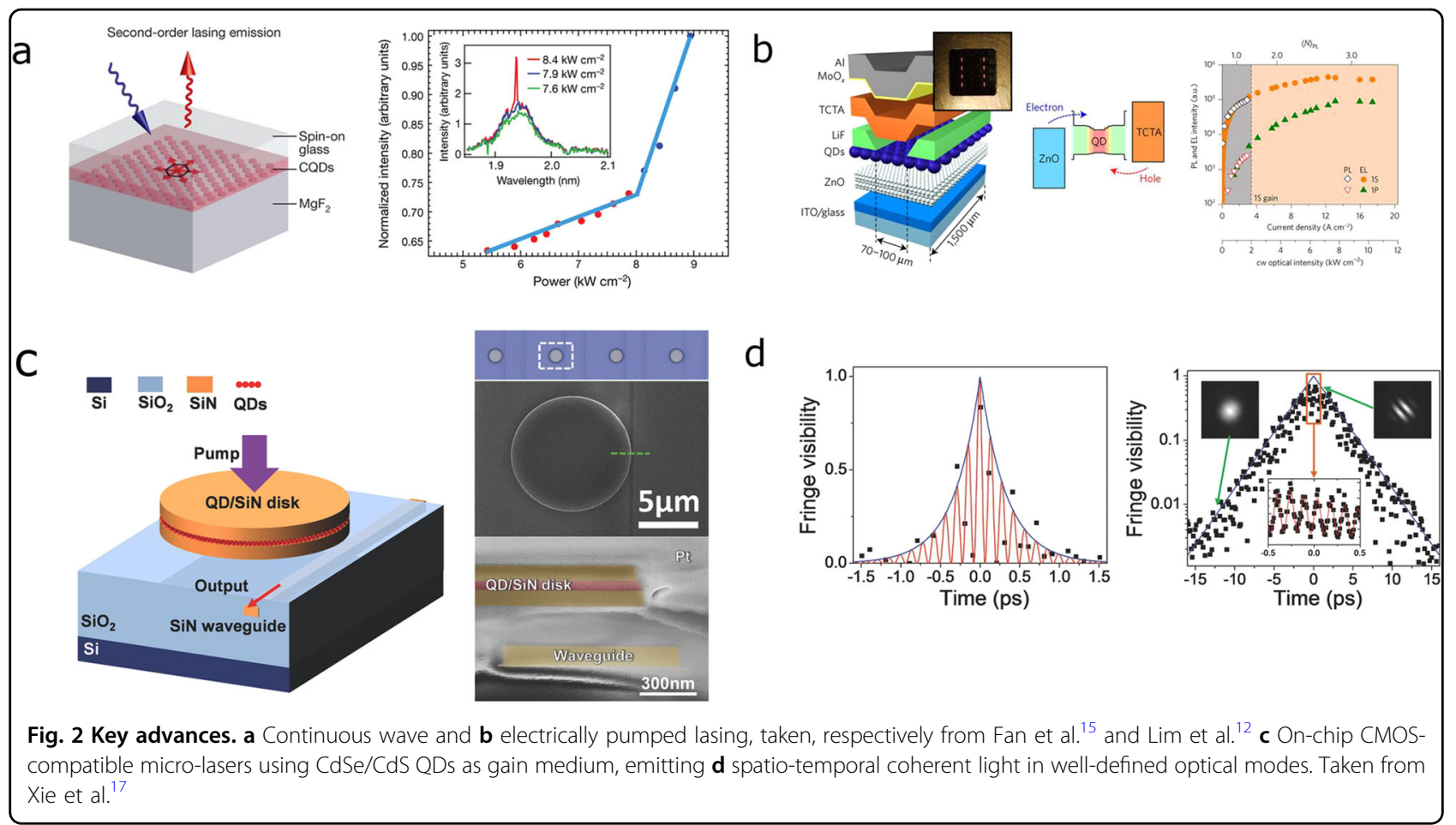

other hand, researchers have explored new materials with a focus on improving the three gain descriptors mentioned above, e.g., by extending the gain lifetime or reducing the threshold occupation.

The ability to pump QD-based lasers with continuous wave $(\mathrm{CW})$ radiation was long sought after and realized by Fan et al., who demonstrated microsecond sustained lasing and true continuous-wave operation ${ }^{14,15}$. Using QDs with suppressed Auger recombination, they showed that proper heat management-involving optimizations of both the active QD layer and the surrounding cavity materials-is key to achieving true CW operation, see Fig. 2a. In particular, these authors used short-chain ligands to improve thermal conductivity in the QD layer and thermally conductive $\mathrm{MgF}_{2}$ substrates to promote heat extraction away from the active QD layer. They demonstrated lasing under $\mathrm{CW}$ excitation with thresholds close to $c a .8 \mathrm{~kW} / \mathrm{cm}^{2}$ using a blue pump laser, a wavelength/ power combination approaching those found in blue laser diodes commonly used for lighting applications. The samples were cooled using a Peltier stage, but nonetheless, reliable operation was achieved for close to half an hour. In 2018, researchers showed that electrically pumped QD light-emitting diodes can achieve a level of excitation comparable to those found in QD lasers. In particular, Lim et al. demonstrated that amplification of incoming red light is feasible at current levels close to a few $\mathrm{A} / \mathrm{cm}^{2}{ }^{16}$. Key to this achievement was the use of a current-focusing architecture, see Fig. 2b, that increased the current density at the level of the QD layer, thus avoiding damage to the delicate organic charge-transport layers.

In addition to improvements in the excitation scheme of QD lasers, researchers also combined CdSe-based QDs with high-quality deterministic cavities that emit light into a well-defined spatially and temporally coherent mode. A most suitable platform to fabricate such cavities is that of integrated silicon photonics, which relies on the fabrication methods of the CMOS industry. This approach allows for high-precision cavity formation, miniaturization, and reliable device performance, and could produce QD lasers in vast numbers. It contrasts with the numerous ad-hoc cavities fabricated by dropcasting ill-defined QD films onto low-quality resonators, such as microstains, beads, and so on, which have little application or upscaling potential. A case example is the work of Xie et al. and Zhu et al.,17 in which a patterning and embedding process was developed to incorporate QDs into silicon-nitride-based microcavities operating in multi- or single-mode operation under quasi-CW conditions, see Fig. 2c. Importantly, this approach makes optimal use of the high material gain provided by dense QD films to realize small footprint $(10-100 \mu \mathrm{m})$ lasers. Furthermore, these microlasers were shown to operate under nanosecond pumping, a timescale that exceeds the bi-exciton lifetime, making them effectively quasi-CW devices. Finally, high-quality-factor cavities such as those fabricated by precision photonic micro-fabrication will 
lead to increased photon lifetimes, which in turn, push the lasing threshold to its theoretical minimum dictated by the gain material's intrinsic properties, see Fig. $1 \mathrm{~b}$.

Even though CdSe core/shell structures can provide long-lived population inversion, $\left\langle N^{*}\right\rangle$ remains close to 1 electron-hole pair per QD and $\tau_{g}$ is limited to $c a .1 \mathrm{~ns}^{3}$. Since these parameters directly affect the pump fluence required to achieve $(\mathrm{CW})$ lasing, several groups have explored new approaches to reduce $\left\langle N^{*}\right\rangle$ and extend $\tau_{\mathrm{g}}$. In particular, research efforts have focused on attaining single-exciton instead of bi-exciton gain by breaking the balance between absorption and stimulated emission that is intrinsic to a single band-edge exciton. We highlight briefly the role of defect-assisted optical gain, doping and type II heterostructures.

Defect-related optical gain seems counterintuitive, but optical transitions related to in-gap defects are quite common in QDs. These trap-related transitions are often connected to empty states for one charge carrier and emit light at energies below the bandgap where no competing absorption is present, see Fig. 3a. Looking back at formula (1), we can write the absorbance of such a system as: $A=2 f(1-\langle N\rangle) A_{g}+f\langle N\rangle A_{g}-(1-f)\langle N\rangle A_{g}$, where $f$ is the occupation factor of the gap state and $A_{g}$ is the absorbance of the gap state transition. Optical gain is achieved at energies where $\left\langle N^{*}\right\rangle=2 f$. Given the Boltzmann-like tail of the distribution function, $\left\langle N^{*}\right\rangle$ can be arbitrarily low. The latter makes such states very interesting for zero-threshold gain, but trap-related transitions usually suffer from low oscillator strengths that cause any induced absorption to overwhelm stimulated emission in most cases. Geiregat et al. showed that in the case of $\mathrm{HgTe}$ QDs, this delicate balance turns in favor of stimulated emission, leading to record-low CW ASE thresholds in the near infrared, albeit with limited material (or modal) gain ${ }^{18}$.

Similarly, doping QDs has brought forward the ability to reduce optical gain thresholds due to a reduction of the ground-state absorption in favor of stimulated emission ${ }^{19}$.

Due to doping, optical amplification can, in principle, be achieved at near-zero occupation since unexcited fully doped QDs, e.g., with 2 electrons in the two-fold degenerate conduction band of CdSe, do not absorb and singly excited QDs always result in stimulated emission. Looking back to equation (1), this implies: $A(E)=-\frac{N_{h}}{g_{h}} A_{0}(E)=-\frac{\langle N\rangle}{4} A_{0}(E)$. As shown in Fig. 3b, this was evidenced by record-low ASE thresholds in the visible spectrum ${ }^{19}$. There are, however, two caveats with this approach. Optical gain originates from singly excited QDs, yet due to the doping, this state is a trion-like state. Though trion Auger rates are more modest than for biexcitons, the increase in inversion $\tau_{g}$ lifetime will be limited. Moreover, the gain coefficient is strongly diluted by a factor $\frac{\langle N\rangle}{4}$, which in the regime of low-excitation density inevitably leads to low material- and modal-gain coefficients.

Finally, Klimov and coworkers showed that the repulsive interactions between excitons found in type II QDs, such as $\mathrm{CdS} / \mathrm{ZnSe}$, can lead to a reduction of the gain threshold to $2 / 3$ and gain lifetimes resembling the single-exciton radiative lifetime, see Fig. $3 \mathrm{c}^{20}$. Though promising, the use of type II QDs in actual lasing architectures has been limited, probably because the type II geometry exposes carriers to the potentially defective surface, and the associated oscillator strength of the gain transition is diluted due to the limited electron-hole overlap. Moreover, it was shown by Bisschop et al. that the eventual material gain in the single-exciton regime amounts to a mere $\frac{1}{g_{e} g_{h}}$, e.g., $1 / 8$ for Cd-based systems, of the total bandedge intrinsic absorption ${ }^{7}$. The latter might explain the limited number of type II lasers reported in the literature.

\section{Progress needed}

With continuous-wave excitation demonstrated for macroscopic lasing cavities, such as those used by Fan et al. ${ }^{13}$, on-chip CW QD microlasers come within reach. Not only are deterministic on-chip cavities more useful to develop applications, the field of integrated photonics has several decades of experience in heat management that can strongly facilitate stable long-term CW operation. Several approaches are easily implementable, such as cladding waveguide structures with low index, highly conductive oxides or fluorides, working on thermally conductive substrates such as sapphire, and so on. Moreover, the near-endless degree of freedom in cavity design with solution-processable and patternable materials, such as QDs, can also inspire more complex lasing architectures, such as neuromorphic networks or random lasers.

Electrically pumped lasers will be crucial for applications in long-haul fiber-based communication and shortrange optical interconnects. Here, a crucial step will be to balance the loss of contact metals with the gain achievable by the QD material. With intrinsic gains of up to $2000 \mathrm{~cm}^{-17}$, it is a reasonable assumption that losses due to metal contacts can be overcome by smart photonic engineering. Focusing architectures similar to those used in the work of Lim et al. (see Fig. 2b) are often found in VCSELs, which are a standard architecture for fibercoupled lasers used in data communication. Alternative architectures for current injection should also be investigated in which the injecting electrodes are kept far away from the active gain layer, and feedback is provided by dielectric mirror pairs. Combining electrical excitation with integrated micro-lasers, e.g., for on-chip interconnects, might prove more challenging, although the design freedom offered by CMOS technology can again add crucial advantages. 

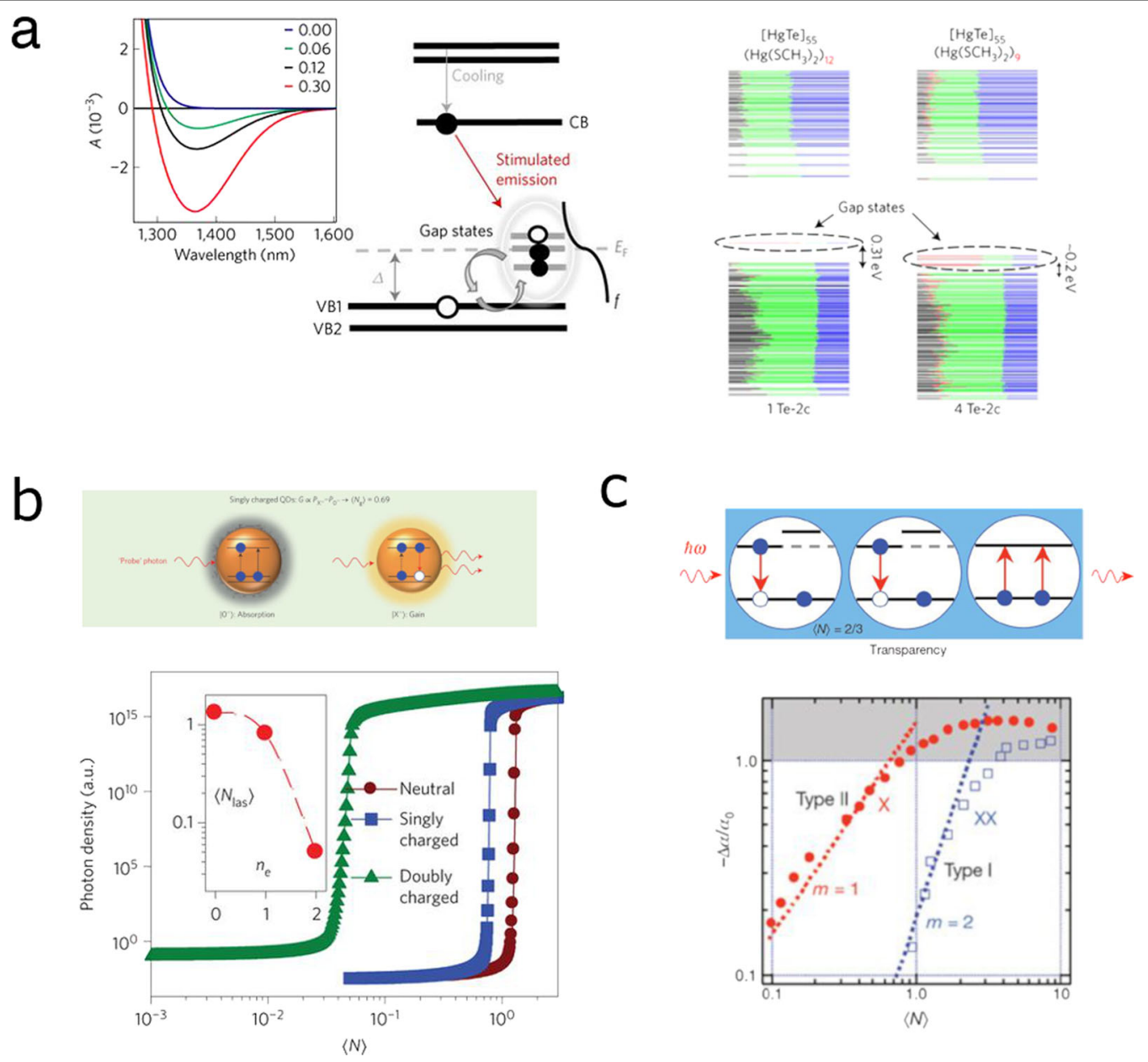

Fig. 3 Single-exciton gain mechanisms. a Defect assisted low threshold gain using HgTe QDs, taken from Geiregat et al. ${ }^{18}$; b Doped Cd-based QDs enable low-threshold amplified spontaneous emission (ASE), taken from Wu et al. ${ }^{19} ;$ c Type II QDs showing thresholds close to 2/3 and prolonged inversion lifetimes, taken from Klimov et al. ${ }^{20}$

Though reports and claims of QD lasing abound, an indepth characterization of the lasing operation itself is mostly missing. Little attention is paid to long-term operational stability, spatial and temporal coherence (see, e.g., Fig. 2d), the ability to modulate the laser output, mode profiles, wall-plug efficiency, and so on. This lack of laser engineering clearly limits progress towards specific applications. A case in point in which engineering becomes relevant is the light output vs. threshold balance. While an increase of the quality factor of the cavities can lower pump thresholds further, see Fig. 1b, such an advance can come at the cost of reduced light output due to increased cavity feedback. Such arguments make clear that QD-based lasers currently require and merit the same level of photonic engineering that pushed epitaxial materials to disruptive applications in the past decades.

In line with this need for proper device engineering, the community also needs to develop a toolbox to reliably report QD gain parameters. Amplified spontaneous emission (ASE) and even variable stripe length (VSL) measurements are very prone to error and fall short of providing a proper comparison between different samples $^{21}$. Here, quantitative femtosecond pump-probe spectroscopy, which measures the material gain $g_{i}(\lambda, t)$ in $\mathrm{cm}^{-1}$ as a function of wavelength and pump-probe delay, offers a useful alternative $e^{7}$. In particular, the material gain is a measure to compare the vastly different samples and material systems available today ${ }^{22}$, and it can be translated to a device context where it yields a good estimate of the modal gain ${ }^{9}$. Moreover, its spectral and transient behaviors provide insights into the gain mechanism and the role of non-radiative Auger processes. Taking this approach, Bisschop et al. could, for example, identify optimal core/shell parameters for $\mathrm{CdSe} / \mathrm{CdS}$ core/ shell QDs, where they found that large-core/thin-shell QDs combinations have the higher material gains, the longer inversion lifetimes and the lower threshold occupation. Material gains up to $2000 \mathrm{~cm}^{-1}$ are accompanied 
by close to $\sim 80$ ps inversion lifetimes and low threshold pump fluences close to $\left\langle N^{*}\right\rangle=1$. This combination was attributed to attractive exciton-exciton interactions, opposite from the common perception that blue-shifted, repulsive, type II bi-excitons are key to efficient gain with QDs. Such high material-gain values, in turn, enabled the fabrication of the previously mentioned microlasers since the modal gain that follows can quite readily overcome typical cavity losses ${ }^{9}$.

\section{Future directions}

Already today, CdSe/CdS QDs offer large gain coefficients under (quasi-)CW pumping and near-unity quantum yields at wavelengths ranging from $\sim 600$ to $\sim 650 \mathrm{~nm}$. In combination with suitable photonic cavities, such optically pumped QD lasers can serve multiple purposes where the low cost of QD synthesis and the versatile deposition and processing will be key differentiators. Onchip optical sensors, for example, can profit greatly from an alternative to the costly epitaxially grown III-V dies required for light emission on a silicon photonics platform $^{23}$. The ability to combine colloidal QDs with nearly every type of substrate could enable flexible lasers for optical bar-codes and wearable optical sensors ${ }^{24}$. The patterning technologies developed also allow for deterministic arrays of possibly sub-wavelength lasers that can enable switchable and spatially multiplexed light emission required for laser displays, $L i F i$, super-resolution imaging, and sub-wavelength sensing ${ }^{25}$. Similar to the success of QDs as down-converting phosphors, one could envisage a QD-based down-conversion laser technology. Indeed, all of the applications mentioned can be steered by readilyavailable blue laser diodes to enable cost-efficient operation. Alternatively, optically pumped light sources can be envisaged that rely on amplified spontaneous emission, in which light is amplified in a single burst without feedback. Such a process provides bright, directional and narrowband radiation, a combination of properties highly sought after in spectroscopy and far-field projection applications. To make such ideas reality in the near future, closer collaborations between materials scientists and device engineers and between academic and industrial research centers is needed. The success of QD displays can be an inspiration and guideline for the latter.

Though cost-effective, small-footprint lasers active in the visible have their merits, several applications require emission at longer wavelengths. Coherence tomography (OCT), telecommunications and trace-gas sensing all need coherent light sources in the near- to mid-wave infrared. Given their bulk bandgap of $3.5 \mu \mathrm{m}$ and $7.3 \mu \mathrm{m}$, InAs and InSb QDs have great potential to reach these exciting wavelength bands. At present, however, synthetic methods do not yield the tuneable core and high-quality core-shell QDs such IR QD lasers will need. A possible direction here can be InAs/CdSe or InAs/InP core/shell systems, both of which feature a straddling band alignment similar to $\mathrm{CdSe} / \mathrm{CdS}$, see Fig. $4 \mathrm{a}^{26}$. Extending the insights in II-VI QDs provided by surface chemistry ${ }^{27}$ and quantitative optical gain studies ${ }^{7}$ to III-V QDs might reveal why, even with the more mature InP-based QDs, no net optical gain has been observed yet.

In addition to a push towards applications and extended-wavelength ranges using $0 \mathrm{D} \mathrm{QDs}$, fundamental research should continue to identify new gain mechanisms and/or potentially more performing solutionprocessable materials. Here, colloidally synthesized twodimensional (2D) materials such as the cadmium chalcogenides and perovskite nanoplatelets ${ }^{28,29}$ stand out as alternative optical gain materials. Reports on continuous wave lasing ${ }^{30}$, small-footprint zero-threshold devices ${ }^{31}$ and disruptive modal gain coefficients ${ }^{32}$ indicate that the optical gain characteristics of CdSe nanoplatelets differ markedly from those of CdSe QDs (see Fig. 4b). Even so, optical gain in nanoplatelets is often discussed in similar terms as QDs, where the word exciton is interchanged. However, excitons in 2D materials differ vastly from electron-hole pairs in the 0D counterparts, and it remains unclear what the exact gain mechanism is in 2D nanoplatelets $^{33}$. Gain in quantum wells, for example, is typically mediated at room temperature by free charge carriers and at cryogenic temperatures by excitonic molecules, i.e., bound quasi-particles consisting of two excitons; the latter give similarly disruptive gain coefficients up to $10^{5} \mathrm{~cm}^{-1}$, comparable to nanoplatelets at room temperature ${ }^{34,35}$. We expect that a proper understanding of the gain mechanism in 2D nanoplatelets will open up new possibilities to use other solutionprocessable excitonic $2 \mathrm{D}$ materials as well, such as transition-metal dichalcogenides or carbon-based nanostructures, to achieve an optical gain at room temperature ${ }^{36,37}$.

Despite the success of $\mathrm{CdSe} / \mathrm{CdS}$ QDs in the 600-650 nm window, achieving similar operation across the whole visible spectrum has proved difficult with these materials. In recent years, solution-processable perovskite materials have emerged as possible alternatives to the more established II-VI colloidal semiconductors, see Fig. $4 \mathrm{c}$ and d. Although quantitative research has indicated that perovskites and their QD morphologies are not intrinsically better gain materials than CdSe/CdS QDs ${ }^{22}$, the wider band-gap tuning, higher thermal conductivity, and improved charge-carrier mobilities can give perovskite nanocrystals an edge to fabricate solutionprocessable lasers across the entire visible spectrum ${ }^{38}$.

\section{Conclusion}

After nearly three decades of nanocrystal research, the field has reached a point where commercially viable 


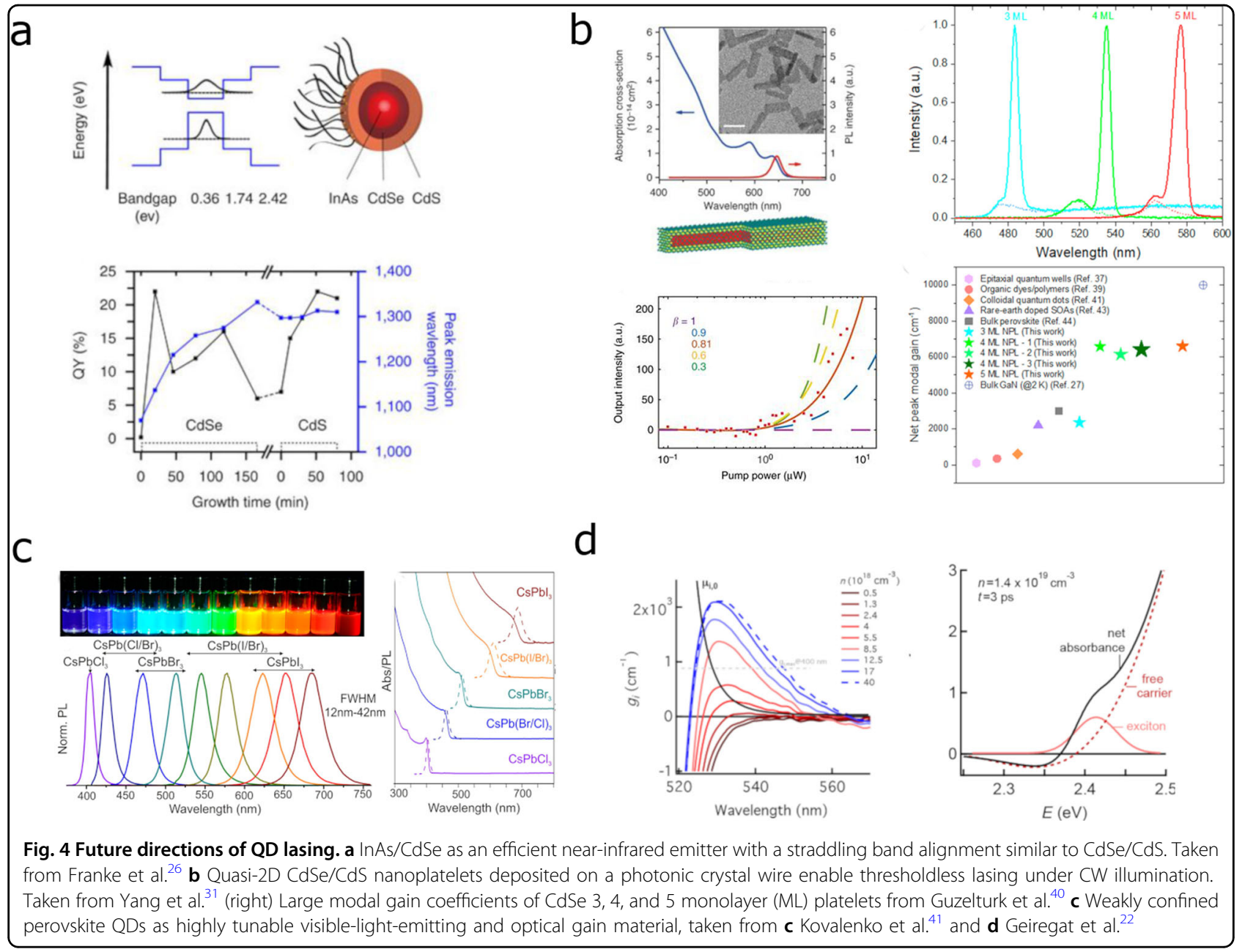

spontaneous and, given a few years, coherent light sources under electrical and optical excitation are within reach. In addition to the established CdSe/CdS QDs, several promising materials exist to address a wider spectral range, increase functionality and attain energy-efficient operation. Described as a future prospect only 4 years ago ${ }^{39}$, $\mathrm{CW}$ operation and electrical excitation have been established today. The quick pace at which QD lasing research advances, even in such short time spans, can only be an inspiration and motivation to current and future researchers and opto-electronic engineers to make those final leaps towards disruptive applications, many of which can benefit our daily lives.

\section{Acknowledgements}

The authors wish to acknowledge A. Houtepen, C. Delerue and I. Moreels for fruitful discussions throughout the years and Ghent University, FWO

Vlaanderen and H2020-MSCA actions for financial support.

\section{Author details}

'Physics and Chemistry of Nanostructures Group, Department of Chemistry, Ghent University, 9000 Gent, Belgium. ${ }^{2}$ Centre for Nano and Bio-photonics,
Ghent University, 9000 Gent, Belgium. ${ }^{3}$ Photonics Research Group, Department of Information Technology, Ghent University, 9000 Gent, Belgium

\section{Conflict of interest}

The authors declare that they have no conflict of interest.

\section{Publisher's note}

Springer Nature remains neutral with regard to jurisdictional claims in published maps and institutional affiliations.

Received: 31 January 2019 Revised: 5 May 2019 Accepted: 29 May 2019. Published online: 9 August 2019

\section{References}

1. Kovalenko, M. V. et al. Prospects of nanoscience with nanocrystals. ACS Nano 9, 1012-1057 (2015).

2. Kagan, C. R., Lifshitz, E., Sargent, E. H. \& Talapin, D. V. Building devices from colloidal quantum dots. Science 353, pii: aac5523 (2016).

3. Pietryga, M. et al. Spectroscopic and device aspects of nanocrystal quantum dots. Chem. Rev. 116, 10513-10622 (2016).

4. Klimov, V. I. et al. Optical gain and stimulated emission in nanocrystal quantum dots. Science 290, 314-317 (2000).

5. $\mathrm{Hu}$, J. et al. Flexible integrated photonics: where materials, mechanics and optics meet. Opt. Mater. Express 3, 3989-3994 (2013). 
6. Ozcelik, D. et al. Optofluidic bioanalysis: fundamentals and applications. Nanophotonics 6, 647-661 (2017).

7. Bisschop, S., Geiregat, P., Aubert, T. \& Hens, Z. The impact of core/shell sizes on the optical gain characteristics of $\mathrm{CdSe} / \mathrm{CdS}$ quantum dots. ACS Nano $\mathbf{1 2}$ 9011-9021 (2018).

8. Klimov, V. Optical nonlinearities and ultrafast carrier dynamics in semiconductor nanocrystals. Phys. Chem. B 104, 6112-6123 (2000).

9. Xie, W. et al. Colloidal quantum dots enabling coherent light sources for integrated silicon-nitride photonics. J. Sel. Top. Quant. Electr. 23, 1-13 (2017).

10. García-Santamaría, F. et al. Suppressed Auger recombination in 'giant' nanocrystals boosts optical gain performance. Nano Lett. 9, 3482-3488 (2009).

11. Park, Y.-S., Bae, W. K., Baker, T., Lim, J. \& Klimov, V. I. Effect of auger recombination on lasing in heterostructured quantum dots with engineered core/ shell interfaces. Nano Lett. 15, 7319-7328 (2015).

12. Lim, J., Park, Y.-S. \& Klimov, V. I. Optical gain in colloidal quantum dots achieved with direct-current electrical pumping. Nat. Mater. 1-41 (2017). https://doi.org/ 10.1038/nmat5011.

13. Fan, F. et al. Continuous-wave lasing in colloidal quantum dot solids enabled by facet-selective epitaxy. Nature 544, 75-79 (2017).

14. Adachi, M. M. et al. Microsecond-sustained lasing from colloidal quantum dot solids. Nat. Commun. 6, 8694 (2015).

15. Fan, F. et al. Continuous-wave lasing in colloical quantum dot solids enabled by facet-selective epitaxy. Nature 544, 75-79 (2017).

16. Lim, J., Park, Y.-S. \& Klimov, V. I. Optical gain in colloidal quantum dots achieved with direct-current electrical pumping. Nat. Mater. 17, $42-49$ (2017).

17. Xie, W. et al. On-Chip Integrated Quantum-Dot Silicon-Nitride Microdisk Lasers. Adv. Mater. 29, 1604866 (2017).

18. Geiregat, P. et al. Continuous-wave infrared optical gain and amplified spontaneous emission at ultralow threshold by colloidal $\mathrm{HgTe}$ quantum dots. Nat. Mater. 17, 35-42 (2017).

19. Wu, K, Park, Y.-S., Lim, J. \& Klimov, V. I. Towards zero-threshold optical gain using charged semiconductor quantum dots. Nat. Nanotechnol. 12, 1140-1147 (2017).

20. Klimov, V. I. et al. Single-exciton optical gain in semiconductor nanocrystals. Nature 447, 441-446 (2007).

21. Negro, L., Bettotti, P. \& Pavesi, L. Applicability conditions and experimental analysis of the variable stripe length method for gain measurements. Opt. Commun. 229, 337-348 (2004)

22. Geiregat, P. et al. Using bulk-like nanocrystals to probe intrinsic optical gain characteristics of inorganic lead halide perovskites. ACS Nano 12, 10178-10188 (2018).

23. Rahim, A. et al. Expanding the silicon photonics portfolio with silicon nitride photonic integrated circuits. J. Light. Technol. 35, 639-649 (2017).
24. Karl, M. et al. Flexible and ultra-lightweight polymer membrane lasers. Nat. Commun. 9, 1525 (2018).

25. Ma, R. \& Oulton, R. F. Applications of nanolasers. Nat. Nanotechnol. 14, 12-22 (2019).

26. Franke, $\mathrm{D}$. et al. Continous injection synthesis of indium arsenide quantum dots emissive in the short-wavelength infrared. Nat. Commun. 7, 1-9 (2016).

27. Houtepen, A. J, Hens, Z., Owen, J. S. \& Infante, I. On the origin of surface traps in colloidal II-VI semiconductor nanocrystals. Chem. Mater. (2016). https://doi. org/10.1021/acs.chemmater.6b04648.

28. Ithurria, S. et al. Colloidal nanoplatelets with two-dimensional electronic structure. Nat. Mater. 10, 936-941 (2011).

29. Pelton, M. Carrier dynamics, optical gain, and lasing with colloidal quantum wells. J. Phys. Chem. C. 122, 10659-10674 (2018).

30. Grim, J. Q. et al. Continuous-wave biexciton lasing at room temperature using solution-processed quantum wells. Nat. Nanotechnol. 9, 891-895 (2014).

31. Yang, Z., Pelton, M., Fedin, I. \& Talapin, D. V. A continuous-wave nanolaser using colloidal quantum wells. Nat. Commun. 8, 143 (2017).

32. Guzelturk, B., Pelton, M., Olutas, M. \& Demir, H. V. Giant Modal Gain Coefficients in Colloidal II-VI Nanoplatelets. Nano Lett. 19, 277-282 (2018).

33. Tomar, R. et al. Charge carrier cooling bottleneck opens up nonexcitonic gain mechanisms in Colloidal CdSe quantum wells. J. Phys. Chem. C. 123, 9640-9650 (2019).

34. Kozlov, V., Kelkar, P., Vertikov, A. \& Nurmikko, A. V. Gain spectroscopy of excitonic molecules and its dynamics in a ZnSe single quantum well. Phys. Rev. B 54, 932-937 (1996)

35. Tomar, R. et al. A charge carrier cooling bottleneck opens up non-excitonic gain mechanisms in colloidal CdSe quantum wells. J. Phys. Chem. C (2019). https://doi.org/10.1063/1.3443634.

36. Wang, G. et al. Colloquium: Excitons in atomically thin transition metal dichalcogenides. Rev. Mod. Phys. 90, 21001 (2018).

37. Gaufrès, E. et al. Optical gain in carbon nanotubes. Appl. Phys. Lett. 96, 231105 (2010).

38. Sutherland, B. R. \& Sargent, E. H. Perovskite photonic sources. Nat. Publ. Gr. 10 295-302 (2016)

39. Nurmikko, A. What future for quantum dot-based light emitters? Nat. Nanotechnol. 10, 1001-1004 (2015).

40. Guzelturk, B., Pelton, M., Olutas, M. \& Demir, H. V. Giant Modal Gain Coefficients in Colloidal II-VI Nanoplatelets. Nano Lett. 19, 277-282 (2018).

41. Kovalenko, M. V., Protesescu, L. \& Bodnarchuk, M. I. Properties and potential optoelectronic applications of lead halide perovskite nanocrystals. Sci. (80-). 750, 745-750 (2017). 